\section{A degron created by SMN2 exon 7 skipping is a principal contributor to spinal muscular atrophy severity}

\author{
Sungchan Cho and Gideon Dreyfuss ${ }^{1}$ \\ Howard Hughes Medical Institute and Department of \\ Biochemistry and Biophysics, University of Pennsylvania \\ School of Medicine, Philadelphia, Pennsylvania 19104, USA
}

Spinal muscular atrophy (SMA) is caused by homozygous survival of motor neurons 1 (SMN1) gene deletions, leaving a duplicate gene, SMN2, as the sole source of SMN protein. However, most of the mRNA produced from SMN2 pre-mRNA is exon 7-skipped $(\sim 80 \%)$, resulting in a highly unstable and almost undetectable protein (SMN $\Delta 7$ ). We show that this splicing defect creates a potent degradation signal (degron; SMN $\Delta 7-D E G$ ) at SMN $\Delta 7$ 's C-terminal 15 amino acids. The S270A mutation inactivates $S M N \Delta 7-D E G$, generating a stable $S M N \Delta 7$ that rescues viability of $S M N$-deleted cells. These findings explain a key aspect of the SMA disease mechanism, and suggest new treatment approaches based on interference with SMN $\Delta 7$-DEG activity.

Supplemental material is available at http://www.genesdev.org.

Received November 11, 2009; revised version accepted January 13, 2010.

Spinal muscular atrophy (SMA) is a common and often fatal motor neuron degenerative disease, and a leading genetic cause of infant mortality (Talbot and Davies 2001; Wirth et al. 2006a; Burnett et al. 2009a). SMA severity corresponds to the degree of functional survival of motor neurons (SMN) protein deficiency. SMN is a ubiquitously expressed protein that plays a critical role in RNA metabolism, and is essential for viability of all cells in eukaryotes (Yong et al. 2004; Neuenkirchen et al. 2008). As part of a large multiprotein complex, the SMN complex, SMN functions in the biogenesis of small nuclear ribonucleoproteins (snRNPs), the major subunits of the spliceosome (Fischer et al. 1997; Liu et al. 1997; Meister et al. 2001; Pellizzoni et al. 2002). Although SMN deficiency manifests itself as a motor neuron disease, its molecular consequences are evident as profound disruptions in RNA metabolism in all tissues tested in an SMA mouse model (Gabanella et al. 2007; Zhang et al. 2008). There are two SMN genes in humans, SMN1 and SMN2, both encoding the same ORF. The vast majority of SMA patients have homozygous $S M N 1$ deletions and are sustained by one or more copies of SMN2. However, due to

[Keywords: Survival of motor neurons $(\mathrm{SMN})$; spinal muscular atrophy (SMA); motor neuron degenerative disease; protein degradation signal (degron); protein stability; pre-mRNA splicing]

${ }^{1}$ Corresponding author.

E-MAIL gdreyfuss@hhmi.upenn.edu; FAX (215) 573-2000.

Article is online at http://www.genesdev.org/cgi/doi/10.1101/gad.1884910. a C/T substitution at position 6 of exon 7 that does not change the encoded amino acid, the splicing of the SMN2 pre-mRNA incurs frequent $(\sim 80 \%)$ exon 7 skipping. This produces an SMN protein (SMN $\Delta 7$ ) that lacks the normal C-terminal 16 amino acids and acquires instead four amino acids, EMLA, encoded by exon 8 (Le et al. 2005). Thus, SMN1 deletions expose the splicing defect of SMN2 and its ineffectiveness in producing full-length normal SMN protein (Wirth et al. 2006a; Cooper et al. 2009). Biochemical experiments in vitro suggested that SMN $\Delta 7$ is not fully functional compared with normal SMN protein, including a diminished oligomerization and binding to protein substrates such as the snRNP Sm proteins (Lorson et al. 1998; Pellizzoni et al. 1999). However, as SMN $\Delta 7$ is extremely unstable and is generally undetectable, a definitive measure of its functional deficit in cells has not been possible. Nevertheless, increased SMN2 copy number correlates with a milder clinical phenotype in SMA patients (Wirth et al. 2006b). For example, severe SMA (type I) patients typically have one or two SMN2 copies, intermediate severity SMA (type II) patients usually have three SMN2 copies, and patients with mild SMA (type III) mostly have three or four SMN2 copies (Feldkotter et al. 2002; Cusco et al. 2006). Furthermore, studies in cells (Wang and Dreyfuss 2001b) suggestedand experiments in SMN-deficient mice demonstratedthat expression of an increasing copy number of SMN $\Delta 7$ cDNA transgenes proportionately lessens SMA severity (Le et al. 2005). This suggests that even a modest SMN $\Delta 7$ increase is beneficial in SMA. With this in mind, our experiments here were designed to determine the cause of SMN $\Delta 7$ instability.

\section{Results and Discussion}

We first established a reporter system that recapitulates the differential stability of full-length SMN and SMN $\Delta 7$ and allows quantitative assessment of SMN $\Delta 7$ 's instability determinants. Luciferase (Luc) reporter proteins consisting of normal SMN or SMN $\Delta 7$ fused to the C terminus of Luc were produced by transfection of the corresponding cDNA constructs in $293 \mathrm{~T}$ cells (Fig. 1). Forty-eight hours after transfection, cells were treated with the protein synthesis inhibitor cycloheximide (CHX), and Luc activity was measured at time intervals of up to $10 \mathrm{~h}$. Consistent with previous reports (Lorson and Androphy 2000), SMN has a half-life of $>8 \mathrm{~h}$, whereas SMN $\Delta 7$ has a half-life of $\sim 3 \mathrm{~h}$. After $10 \mathrm{~h}$ of CHX chase, there was three times more SMN than SMN $\Delta 7$. Several constructs were prepared to determine the role of the C-terminal sequence of SMN $\Delta 7$ in this protein's instability. Deletion of the C-terminal EMLA from SMN $\Delta 7$ (SMN $\Delta 7 \Delta$ EMLA) increased the half-life of SMN $\Delta 7$ by twofold (Fig. 1B), and a further deletion of the YG box (SMN $\Delta 7 \Delta \mathrm{YG}$ ), a conserved tyrosine/glycine-rich motif in divergent SMNs (Talbot et al. 1997) that is essential for SMN oligomerization (Pellizzoni et al. 1999), also had the same effect. These results suggest that EMLA and the YG box are major contributors to SMN $\Delta 7^{\prime}$ 's instability. Importantly, YG + EMLA alone was sufficient to cause dramatic instability of Luc that is similar to that of SMN $\Delta 7$. Neither YG nor EMLA alone was sufficient for full destabilization activity (Fig. 1B). N-terminal deletions 
A

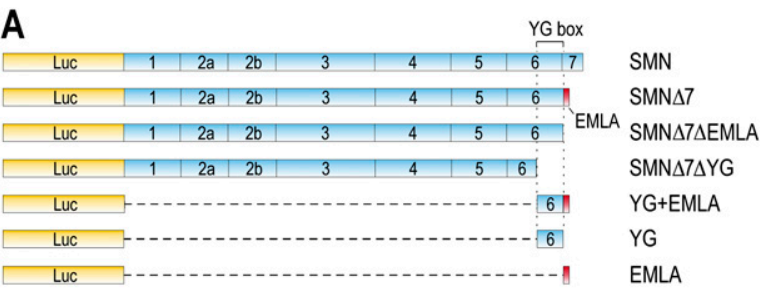

B

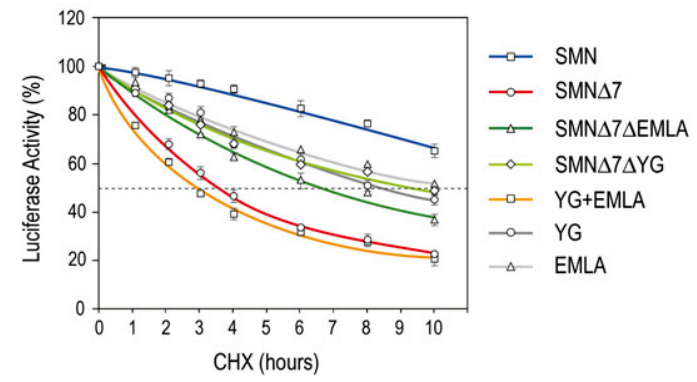

Figure 1. Delineation of YG + EMLA as a protein destabilization sequence in SMN $\Delta 7$. (A) Schematic diagram of Luc-fused SMN and a series of deletion constructs used for quantitative measurement of protein stability. Shown are SMN exon structures. YG box denotes the tyrosine/glycine (YG)-rich sequences in exon 6 of SMN. The EMLA sequence encoded by exon 8 is depicted by the red box at the C-terminal end of SMN $\Delta 7$. (B) $293 \mathrm{~T}$ cells were transfected with plasmids expressing Luc-SMN, Luc-SMN $\Delta 7$, and the indicated deletion constructs. Forty-eight hours after transfection, the cells were treated with CHX $(0.1 \mathrm{mg} / \mathrm{mL})$ for various times as indicated, and then assayed for luciferase activity. Luc activity at each time point was calculated by comparison with those at time 0 , which was set to $100 \%$. Fifty percent activity is indicated by the gray dotted line. Error bars represent standard deviation (SD) from three independent experiments.

in the YG box decreased the destabilizing activity of YG + EMLA (data not shown). These data indicate that YG + EMLA, corresponding to SMN $\Delta 7$ amino acids $268-282$, is the minimal sequence required for full SMN $\Delta 7$ destabilization, and is both necessary and sufficient to trigger rapid degradation of a heterologous protein.

As a further test of this conclusion, we fused YG + EMLA to another reporter, GFP, and expressed this protein as well as GFP as a control in 293 T cells. The GFP signal from GFP-YG + EMLA, as determined by Western blots, showed a gradual decrease after treatment with CHX (Fig. 2A). GFP-YG + EMLA protein decreased faster than GFP-NS (nonspecific sequence), and the half-life of GFP-YG + EMLA was about half that of GFP-NS. These results suggest that $Y G+$ EMLA functions as a protein degradation sequence. For comparison, we tested the destabilizing activity on the same reporter of YG + EMLA and an optimized PEST, a potent and well-characterized protein destabilizing signal ( $\mathrm{Li}$ et al. 1998). YG + EMLA had a similar effect to that of the genetically improved PEST (Fig. 2B). Furthermore, SMN Exon6 + EMLA, which is the same size as the 41-amino-acid PEST sequence, conferred similar instability. As this optimized PEST sequence has about half the half-life of the natural one (Li et al. 1998), YG + EMLA could be estimated to have similar or stronger destabilization activity than that of the natural PEST sequence, and Exon6 + EMLA is about twice as strong. These data demonstrate that YG + EMLA is a highly potent and transferable protein degradation signal (degron), which we term SMN $\Delta 7$-DEG, for SMN $\Delta 7$ degron. Addition of five amino acids to the C-terminal end of EMLA (SMN $\Delta 7+5 \mathrm{aa}$ ) caused SMN $\Delta 7$ stabilization, indicating that SMN $\Delta 7-\mathrm{DEG}$ must be exposed at the $\mathrm{C}$ terminus of the protein for activity (Fig. 2B). This is consistent with the observation that several additional amino acids, which can be effected by aminoglycosideforced translational read-through, enhanced SMN $\Delta 7$ stability and functionality (Mattis et al. 2008; Heier and DiDonato 2009).

SMN was shown previously to be degraded by the proteasome (Chang et al. 2004; Burnett et al. 2009b). To determine if SMN $\Delta 7$ is also degraded by this system, cells expressing Luc-SMN $\Delta 7$ were treated with proteasome inhibitors (MG132 and Lactacystin) for $5 \mathrm{~h}$ in the presence of CHX. CHX treatment alone resulted in a $60 \%$ decrease in signal, but a much smaller decrease was seen in the presence of proteasome inhibitors MG132 and Lactacystin (Supplemental Fig. 1). Inhibitors of other proteolytic activities-such as lysosomal proteases, autophagy, and calpain $\left(\mathrm{NH}_{4} \mathrm{Cl}, 3\right.$-methyladenine, and calpeptin, respectively)-had no effect. These data demonstrate that SMN $\Delta 7$ is degraded by the proteasome.

To identify specific residues in the SMN $\Delta 7-\mathrm{DEG}$ that are important for its activity, we performed mutagenesis of the YG box, converting every second residue to alanine in the context of full-length SMN $\Delta 7$, and determined the half-life of each in $293 \mathrm{~T}$ cells. Of seven mutations tested, S270A produced the most striking effect, reversing the destabilizing activity of the SMN $\Delta 7-D E G$ (Fig. 3A). To confirm that S270A stabilizes SMN $\Delta 7$, HA-tagged proteins SMN, SMN $\Delta 7$, and SMN $\Delta 7^{\text {S270A }}$ were expressed in $293 \mathrm{~T}$ cells for $24 \mathrm{~h}$, and then treated with the proteasome
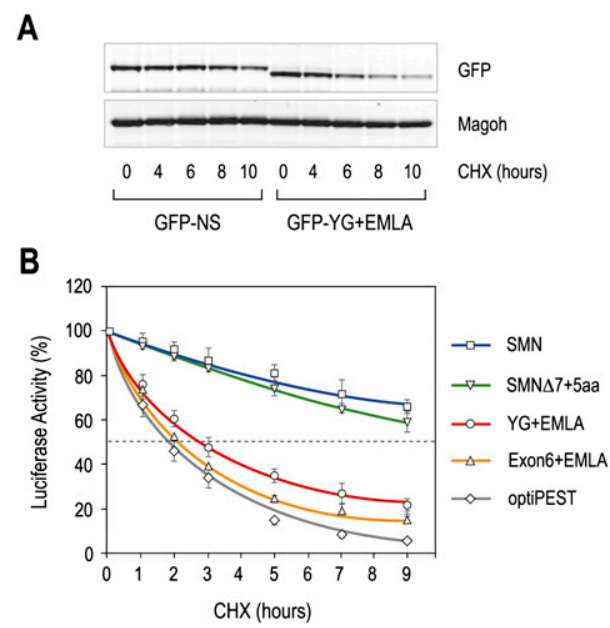

Figure 2. The $\mathrm{C}$ terminus of $\mathrm{SMN} \Delta 7, \mathrm{YG}+\mathrm{EMLA}$, is a strong protein destabilizing signal (degron). (A) Plasmids expressing GFPYG + EMLA or GFP-NS (nonspecific sequence) were transfected into $293 \mathrm{~T}$ cells. Twenty-four hours after transfection, the cells were treated with $\mathrm{CHX}(0.1 \mathrm{mg} / \mathrm{mL})$ for various times as indicated. GFP fusion proteins were detected by Western blot using anti-GFP antibody, and Magoh was used as a loading control. $(B)$ Comparison of YG + EMLA and Exon6 + EMLA of SMN $\Delta 7$ with an optimized protein-destabilizing element (optiPEST). Shown also are Luc-SMN and Luc-SMN $\Delta 7$ containing an additional five amino acids at the C-terminal end (SMN $\Delta 7+5$ aa). Luc activities were measured as in Figure 1. Error bars represent SDs from three independent experiments. 
A

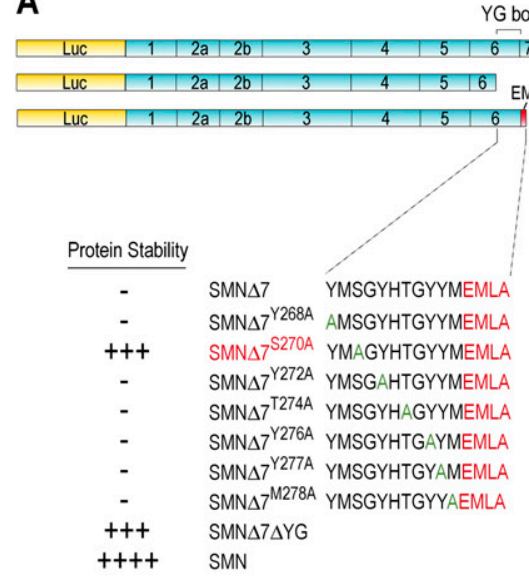

B

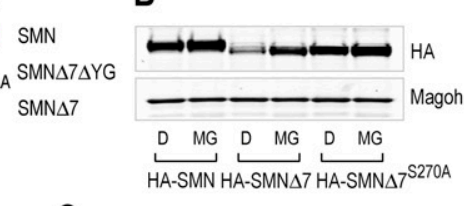

C

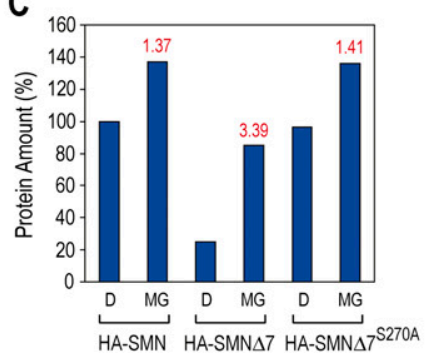

D
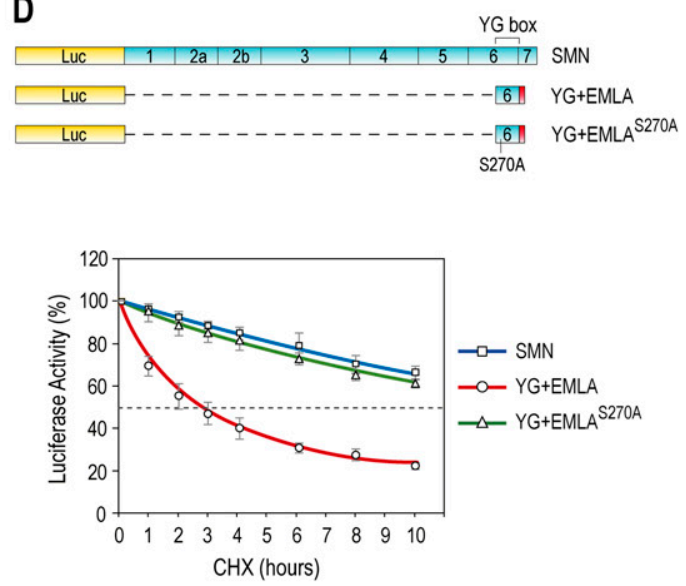

Figure 3. S270 is critical for the activity of the SMN $\Delta 7-D E G$ through YG + EMLA. (A) Seven residues in the YG box were each mutated to alanine as indicated. All constructs had Luc fusions, and Luc activity was assayed as in Figure 1. $(B)$ HA-tagged SMN, SMN $\Delta 7$, and SMN $\Delta 7^{\mathrm{S} 270 \mathrm{~A}}$ were expressed in $293 \mathrm{~T}$ cells for $24 \mathrm{~h}$, and then cells were treated with DMSO (D) or $10 \mu \mathrm{M}$ MG132 (MG) for $16 \mathrm{~h}$. Fusion proteins were monitored by Western blot using an anti-HA tag antibody, and Magoh was used as a loading control. (C) HA-tagged proteins in $B$ were quantified and compared with HA-SMN without MG132 treatment, which was set to $100 \%$. The fold change of each fusion protein amount upon MG132 treatment is indicated in red above the column. $(D)$ All constructs had Luc fusions, and Luc activity was assayed as in Figure 1. Error bars represent SDs from three independent experiments.

inhibitor MG132 for $16 \mathrm{~h}$. The levels of the tagged SMN proteins were then monitored by Western blots using anti-HA antibody (Fig. 3B). As expected, the amount of SMN $\Delta 7$ without MG132 treatment was much lower than that of SMN. However, the amount of SMN $\Delta 7^{\text {S270A }}$ was similar to that of normal SMN, indicating an almost complete restoration of stability by S270A mutation. MG132 caused a dramatic increase in the amount of SMN $\Delta 7$ ( $\sim 3.4$-fold), but only a moderate effect on SMN and SMN $\Delta 7^{\text {S270A }}$ (Fig. 3C). Therefore, the S270A mutation limits the proteosome degradation of SMN $\Delta 7$ and increases its stability very significantly. We further tested the effect of S270A in the context of SMN $\Delta 7$-DEG alone. S270A mutation strongly increased the stability of LucYG + EMLA to a level similar to that of SMN (Fig. 3D). These data indicate that the enhancement of stability of SMN $\Delta 7$ by the S270A mutation occurs through SMN $\Delta 7$ DEG.

To determine whether $\operatorname{SMN} \Delta 7^{\mathrm{S} 270 \mathrm{~A}}$ is a functional SMN protein, we used a previously established cell system, the S5 cell line, to ask if it could rescue the viability of SMN-depleted cells. S5 is derived from chicken DT40 cells in which the endogenous chicken $S M N$ gene is disrupted by homologous recombination and SMN protein is expressed exogenously from a cDNA under a tetracycline-repressible promoter (Wang and Dreyfuss 2001a). Upon depletion of chicken SMN (cSMN), S5 cell growth arrests at $72 \mathrm{~h}$ and cell death occurs. It is therefore useful to assess the physiological functionality of SMN mutants in this cell system by monitoring cell viability after turning off cSMN cDNA expression and simultaneously expressing exogenous SMN mutants of interest. To do so, we constructed recombinant retroviruses expressing $\mathrm{SMN}, \mathrm{SMN} \Delta 7$, or SMN $\Delta 7^{\mathrm{S} 270 \mathrm{~A}}$ and transduced S5 cells. One week after repression of cSMN expression by tetracycline $(1 \mu \mathrm{g} / \mathrm{mL})$, there was a very clear difference in viable cell number among three samples (Fig. 4A,B). As expected (Wang and Dreyfuss 2001b), while SMN rescued the viability of S5 cells, SMN $\Delta 7$ did not. Importantly, $\mathrm{SMN} \Delta 7^{\mathrm{S} 270 \mathrm{~A}}$ also rescued S5 cells to a similar extent as SMN. The two rescued cell lines expressed a similar level of SMN protein (Fig. 4C). Since the deficiency in functional SMN protein is correlated directly with snRNP assembly defects in cells of SMA patients (Wan et al. 2005), we next examined whether SMN $\Delta 7^{\text {S270A }}$ is active in snRNP assembly as a further measure of functionality. Extracts
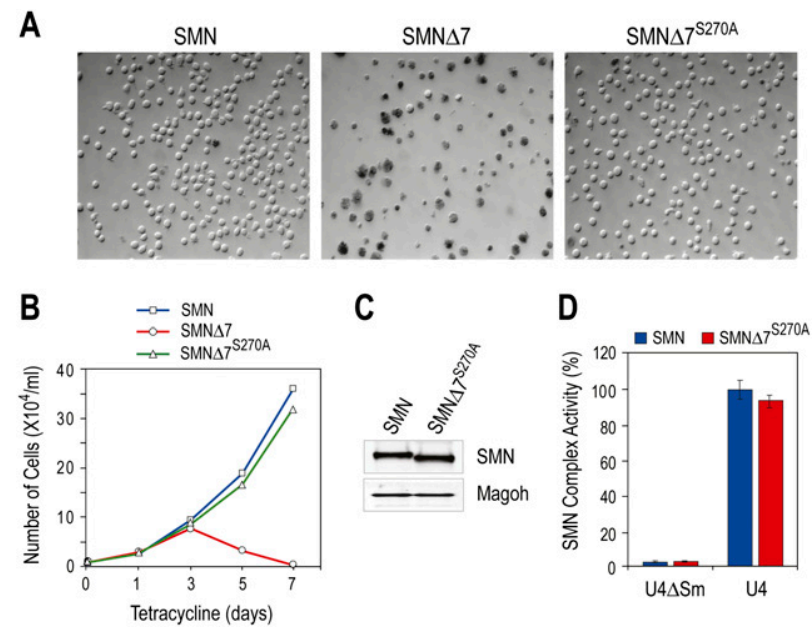

Figure 4. SMN $\Delta 7^{\mathrm{S} 270 \mathrm{~A}}$ rescues $\mathrm{SMN}$-deficient cells and is functional in snRNP assembly. (A) S5 cells were cultured in the presence of tetracycline $(1 \mu \mathrm{g} / \mathrm{mL})$ to deplete endogenous SMN, and were infected with retroviruses expressing SMN, SMN $\Delta 7$, or SMN $\Delta 7^{\mathrm{S} 270 \mathrm{~A}}$. One week after tetracycline addition, cells were stained with Trypan blue and visualized by DIC microscopy. (B) Cell growth as in $A$ was measured by monitoring the number of live cells at the indicated time points following tetracycline addition. (C) Western blots of SMN protein in rescued cells (10 d after tetracycline addition). (D) Cytoplasmic extracts from rescued cells were assayed for snRNP assembly on U4 snRNA in vitro, using U4ASm RNA as a control. 
from cells expressing SMN and SMN $\Delta 7^{\mathrm{S} 270 \mathrm{~A}}$ were prepared, and their snRNP assembly activity was determined by measuring the amount of Sm protein cores that form on biotinylated snRNA substrate captured on streptavidin beads (Wan et al. 2005). Sm cores are the major constituents of snRNPs whose assembly on snRNAs depends on the SMN complex. As shown in Figure 4D, both cell lines showed similar activity. These data indicate that SMN $\Delta 7^{\mathrm{S} 270 \mathrm{~A}}$ is a functional protein similar to normal SMN in S5 cells. We conclude that the instability of $\mathrm{SMN} \Delta 7$ conferred by SMN $\Delta 7-\mathrm{DEG}$ is a principal contributor to the deleterious phenotype of exon 7 skipping, and that S270A substitution in SMN $\Delta 7$ abrogates the degron activity, thereby restoring the function of SMN.

Several diverse classes of degrons that target proteins to various degradation pathways have been described. Most noted are $\mathrm{N}$ degrons comprised of destabilizing $\mathrm{N}$-terminal residues, $\mathrm{C}$-terminal determinants containing relatively unstructured hydrophobic residues, and phospho-degrons that are modulated by the phosphorylation status of their serine/threonine residues in response to cell signaling (Parsell et al. 1990; Ravid and Hochstrasser 2008). The short-lived tumor suppressor protein PTEN's stability depends on a 50-amino-acid C-terminal tail that is phosphorylated at specific serine/threonine residues (Vazquez et al. 2000). Interestingly, while many of the residues of the SMN $\Delta 7$-DEG could be substituted by alanines without loss of degron function, S270 is critical for the destabilizing function. It is therefore possible that S270 is phosphorylated, and that this regulates the SMN $\Delta 7$-DEG activity. However, phosphorylation site analysis by NetPhos did not reveal strong candidate kinases for it. SMN $\Delta 7$-DEG has no obvious sequence similarity with the known degrons and, thus, represents a novel protein-destabilizing element. Protein database searches did not identify other known proteins containing sequences highly similar to SMN $\Delta 7-D E G$.

The reduced oligomerization efficiency of SMN $\Delta 7$ has been suggested recently to account for its instability (Burnett et al. 2009b). Indeed, intermolecular SMN oxidative cross-linking provided direct evidence that SMN is oligomeric in cells (Wan et al. 2008). Oligomerization is likely to be important for SMN function, and also to contribute to its stability. However, although SMN oligomerization correlated with its stability, this did not explain the intrinsic instability of SMN $\Delta 7$. Our findings show that attachment of SMN $\Delta 7$-DEG to monomeric protein reporters (Luc and GFP) triggered their rapid degradation, indicating that lack of oligomerization is not the major cause of SMN $\Delta 7$ 's instability. Loss of oligomerization capacity and other possible deficits as a result of deletion of the peptide encoded by exon 7 may result in an SMN protein that is functionally suboptimal. However, the detrimental effect of exon 7 skipping does not arise primarily from deletion of a functionally essential domain, but from the creation of a positively acting and potent degron that causes severe deficiency of $\mathrm{SMN} \Delta 7$ protein.

Given the ability of S270A mutation to restore SMN $\Delta 7$ 's stability and complement SMN loss of function, it is reasonable to predict that polymorphisms that inactivate SMN $\Delta 7-D E G$, such as at S270, would result in a milder SMA phenotype than the genotype predicts based on SMN2 copy number in SMN1-deleted individ- uals. Our finding with $\mathrm{SMN} \Delta 7^{\mathrm{S} 270 \mathrm{~A}}$ indicates that $\mathrm{SMN} \Delta 7$ is a functional SMN protein, and that its stabilization could prevent or lessen SMA severity. We suggest that interfering with SMN $\Delta 7$-DEG activity could be an effective approach for mitigating its deficiency as a potential treatment for SMA. Although the inhibitor studies suggest that the degradation of SMN $\Delta 7$ likely occurs in the proteasome, general inhibition of proteasome activity would be very toxic, particularly in the long-term treatment that SMA would be expected to require. A targeted inhibition of the factors that mediate the SMN $\Delta 7-D E G-$ dependent degradation should provide a more specific therapeutic approach, and their identification will be of great interest for SMA therapy.

SMA is thus the result of a fateful chain of events. Homozygous SMN1 deletion is a cause of SMA only because it exposes the splicing defect of SMN2. We argue that the splicing defect in SMN2 causes SMN deficiency because it fortuitously creates a degron. The degron is a key to SMA, as it is the most direct cause of SMN deficiency, which then results in major perturbations in RNA metabolism.

\section{Materials and methods}

\section{Plasmid construction and generation of mutations}

To construct plasmids expressing Luc-fused proteins, the Luc gene was cloned into pcDNA3.1 vector at HindIII/KpnI sites, and then DNA fragments encoding full-length wild-type human $\operatorname{SMN}, \operatorname{SMN} \Delta 7$, several deletion mutants of SMN $\Delta 7$, and optiPEST were inserted into the $\mathrm{KpnI} / \mathrm{XhoI}$ sites. SMN $\Delta 7$ mutants with a single amino acid change were generated by mutating residues in YG + EMLA to alanine by QuickChange site-directed mutagenesis kit (Stratagene). Plasmid expressing GFP-YG + EMLA was constructed by inserting a DNA fragment encoding YG + EMLA into pEGFP vector (Clontech) at KpnI/BamHI sites. Plasmids expressing HASMNs were constructed by inserting DNA fragments encoding HA-tagged SMN, SMN $\Delta 7$, and SMN $\Delta 7^{\text {S270A }}$ into the BamHI/Xhol sites of pcDNA3 vector. To generate retroviral plasmids to express SMNs in S5 cells, DNA fragments encoding SMN, SMN $\Delta 7$, and SMN $\Delta 7^{\text {S270A }}$ were cloned into the EcoRI/XhoI sites of pMX vector as described (Wang and Dreyfuss 2001a).

\section{Assays for protein stability}

Luc- and GFP-based assays were performed as described in the legends for Figures 1 and 2A, respectively. Luc activities were measured using OneGlo reagent (Promega).

\section{Rescue of S5 cell viability}

S5 cells were maintained and infected with retroviruses expressing SMN, SMN $\Delta 7$, and SMN $\Delta 7^{\text {S270A }}$ as described (Wang and Dreyfuss 2001a).

\section{SMN complex activity assay}

Cytoplasmic extracts from rescued S5 cells were prepared and assayed for snRNP assembly in vitro as described (Wan et al. 2005).

\section{Antibodies}

Mouse monoclonal antibodies anti-SMN (62E7) and anti-Magoh (18G12) were used as described previously (Wan et al. 2005). Rabbit polyclonal antibodies anti-HA (Santa Cruz Biotechnologies) and anti-GFP (Santa Cruz Biotechnologies) were used as recommended by the manufacturer.

\section{Acknowledgments}

We thank the members of our laboratory, especially Dr. Lili Wan, for helpful discussions and comments on this manuscript. This work was 
supported by the Association Française Contre les Myopathies (AFM). G.D. is an Investigator of the Howard Hughes Medical Institute.

\section{References}

Burnett BG, Crawford TO, Sumner CJ. 2009a. Emerging treatment options for spinal muscular atrophy. Curr Treat Options Neurol 11: 90-101.

Burnett BG, Munoz E, Tandon A, Kwon DY, Sumner CJ, Fischbeck KH. 2009b. Regulation of SMN protein stability. Mol Cell Biol 29: 11071115 .

Chang HC, Hung WC, Chuang YJ, Jong YJ. 2004. Degradation of survival motor neuron (SMN) protein is mediated via the ubiquitin/proteasome pathway. Neurochem Int 45: 1107-1112.

Cooper TA, Wan L, Dreyfuss G. 2009. RNA and disease. Cell 136: 777793.

Cusco I, Barcelo MJ, Rojas-Garcia R, Illa I, Gamez J, Cervera C, Pou A, Izquierdo G, Baiget M, Tizzano EF. 2006. SMN2 copy number predicts acute or chronic spinal muscular atrophy but does not account for intrafamilial variability in siblings. J Neurol 253: 21-25.

Feldkotter M, Schwarzer V, Wirth R, Wienker TF, Wirth B. 2002. Quantitative analyses of SMN1 and SMN2 based on real-time lightCycler PCR: Fast and highly reliable carrier testing and prediction of severity of spinal muscular atrophy. Am J Hum Genet 70: 358-368.

Fischer U, Liu Q, Dreyfuss G. 1997. The SMN-SIP1 complex has an essential role in spliceosomal snRNP biogenesis. Cell 90: 1023-1029.

Gabanella F, Butchbach ME, Saieva L, Carissimi C, Burghes AH, Pellizzoni L. 2007. Ribonucleoprotein assembly defects correlate with spinal muscular atrophy severity and preferentially affect a subset of spliceosomal snRNPs. PLoS One 2: e921. doi: 10.1371/journal.pone. 0000921.

Heier CR, DiDonato CJ. 2009. Translational readthrough by the aminoglycoside geneticin (G418) modulates SMN stability in vitro and improves motor function in SMA mice in vivo. Hum Mol Genet 18: 1310-1322.

Le TT, Pham LT, Butchbach ME, Zhang HL, Monani UR, Coovert DD, Gavrilina TO, Xing L, Bassell GJ, Burghes AH. 2005. SMN $\Delta 7$, the major product of the centromeric survival motor neuron (SMN2) gene, extends survival in mice with spinal muscular atrophy and associates with full-length SMN. Hum Mol Genet 14: 845-857.

Li X, Zhao X, Fang Y, Jiang X, Duong T, Fan C, Huang CC, Kain SR. 1998. Generation of destabilized green fluorescent protein as a transcription reporter. J Biol Chem 273: 34970-34975.

Liu Q, Fischer U, Wang F, Dreyfuss G. 1997. The spinal muscular atrophy disease gene product, SMN, and its associated protein SIP1 are in a complex with spliceosomal snRNP proteins. Cell 90: 1013-1021.

Lorson CL, Androphy EJ. 2000. An exonic enhancer is required for inclusion of an essential exon in the SMA-determining gene SMN. Hum Mol Genet 9: 259-265.

Lorson CL, Strasswimmer J, Yao JM, Baleja JD, Hahnen E, Wirth B, Le T, Burghes AH, Androphy EJ. 1998. SMN oligomerization defect correlates with spinal muscular atrophy severity. Nat Genet 19: 63-66.

Mattis VB, Bowerman M, Kothary R, Lorson CL. 2008. A SMN $\Delta 7$ readthrough product confers functionality to the SMN $\Delta 7$ protein. Neurosci Lett 442: 54-58.

Meister G, Buhler D, Pillai R, Lottspeich F, Fischer U. 2001. A multiprotein complex mediates the ATP-dependent assembly of spliceosomal U snRNPs. Nat Cell Biol 3: 945-949.

Neuenkirchen N, Chari A, Fischer U. 2008. Deciphering the assembly pathway of Sm-class U snRNPs. FEBS Lett 582: 1997-2003.

Parsell DA, Silber KR, Sauer RT. 1990. Carboxy-terminal determinants of intracellular protein degradation. Genes \& Dev 4: 277-286.

Pellizzoni L, Charroux B, Dreyfuss G. 1999. SMN mutants of spinal muscular atrophy patients are defective in binding to snRNP proteins. Proc Natl Acad Sci 96: 11167-11172.

Pellizzoni L, Yong J, Dreyfuss G. 2002. Essential role for the SMN complex in the specificity of snRNP assembly. Science 298: 1775-1779.

Ravid T, Hochstrasser M. 2008. Diversity of degradation signals in the ubiquitin-proteasome system. Nat Rev Mol Cell Biol 9: 679-690.

Talbot K, Davies KE. 2001. Spinal muscular atrophy. Semin Neurol 21: $189-197$.

Talbot K, Ponting CP, Theodosiou AM, Rodrigues NR, Surtees R, Mountford R, Davies KE. 1997. Missense mutation clustering in the survival motor neuron gene: A role for a conserved tyrosine and glycine rich region of the protein in RNA metabolism? Hum Mol Genet 6: 497-500.

Vazquez F, Ramaswamy S, Nakamura N, Sellers WR. 2000. Phosphorylation of the PTEN tail regulates protein stability and function. Mol Cell Biol 20: 5010-5018.

Wan L, Battle DJ, Yong J, Gubitz AK, Kolb SJ, Wang J, Dreyfuss G. 2005. The survival of motor neurons protein determines the capacity for snRNP assembly: Biochemical deficiency in spinal muscular atrophy. Mol Cell Biol 25: 5543-5551.

Wan L, Ottinger E, Cho S, Dreyfuss G. 2008. Inactivation of the SMN complex by oxidative stress. Mol Cell 31: 244-254.

Wang J, Dreyfuss G. 2001a. A cell system with targeted disruption of the SMN gene: Functional conservation of the SMN protein and dependence of Gemin2 on SMN. J Biol Chem 276: 9599-9605.

Wang J, Dreyfuss G. 2001b. Characterization of functional domains of the SMN protein in vivo. I Biol Chem 276: 45387-45393.

Wirth B, Brichta L, Hahnen E. 2006a. Spinal muscular atrophy: From gene to therapy. Semin Pediatr Neurol 13: 121-131.

Wirth B, Brichta L, Schrank B, Lochmuller H, Blick S, Baasner A, Heller R. 2006b. Mildly affected patients with spinal muscular atrophy are partially protected by an increased SMN2 copy number. Hum Genet 119: $422-428$.

Yong J, Wan L, Dreyfuss G. 2004. Why do cells need an assembly machine for RNA-protein complexes? Trends Cell Biol 14: 226-232.

Zhang Z, Lotti F, Dittmar K, Younis I, Wan L, Kasim M, Dreyfuss G. 2008. SMN deficiency causes tissue-specific perturbations in the repertoire of snRNAs and widespread defects in splicing. Cell 133: 585-600. 


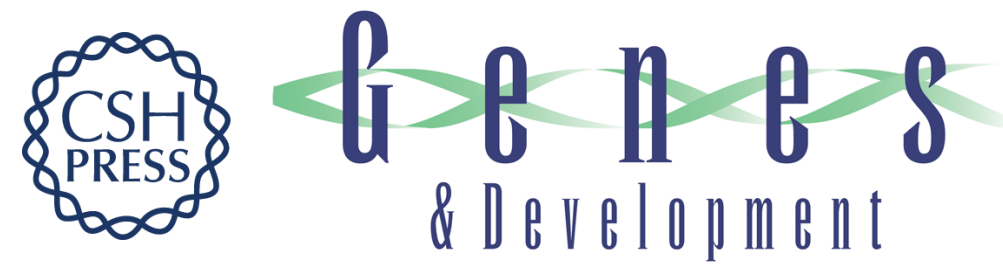

\section{A degron created by SMN2 exon 7 skipping is a principal contributor to spinal muscular atrophy severity}

Sungchan Cho and Gideon Dreyfuss

Genes Dev. 2010, 24:

Access the most recent version at doi:10.1101/gad.1884910

Supplemental
Material http://genesdev.cshlp.org/content/suppl/2010/02/18/24.5.438.DC1

References This article cites 32 articles, 9 of which can be accessed free at: http://genesdev.cshlp.org/content/24/5/438.full.html\#ref-list-1

License

Email Alerting

Receive free email alerts when new articles cite this article - sign up in the box at the top Service right corner of the article or click here.

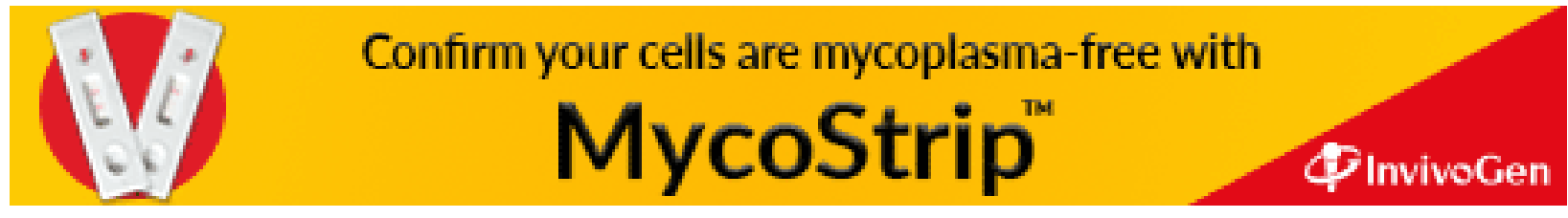

